\title{
Study of Prevalence of GUDs in STD Clinic Attendees of A Tertiary Care Hospital
}

\author{
DSS Srinivas Prasad ${ }^{1}$, TV Narasimharao ${ }^{2}, \mathrm{~S} \mathrm{Nageswaramma}^{3}$. \\ Department Of DVL, Guntur Medical College, Guntur, Andhra Pradesh, India.
}

\begin{abstract}
Introduction: Sexually Transmitted Diseases (STDs) are a burden to the individual, his family and the community as the patients are mostly from the working age group. Genital Ulcer Diseases (GUDs) are a type of manifestation of STDs. GUDs are commonly caused by Syphilis, Chancroid, Donovanosis, LymphoGranulomaVenereum (LGV) and Genital Herpes. It is now clearly understood that GUDs facilitate Human Immunodeficiency Virus (HIV) transmission and vice versa.

Objectives: In this context a study was conducted by the department of DermatoVenereoLeprology (DVL) to study the prevalence of STDs in patients attending STD clinic over a period of one year.

Methodology: All the patients attending the STD Clinic from 01-04-2015 to 31-03-2016 were enrolled in the study and examined for GUDs. After necessary investigations appropriate diagnosis of the GUD was made. The results were analysed.

Results: The prevalence of GUDs in total STD clinic attendees is $2.57 \%$. Among the total STD clinic male attendees, the prevalence of GUDs is $6.92 \%$ while it is only $1.12 \%$ in the total female attendees. Genital herpes was the leading cause of GUDs in both male and female patients in our study.

Conclusion: The prevalence of GUDs in the STD clinic attendees is decreasing which is a welcome sign but at the same time, genital herpes is emerging as the leading cause of GUDs. This should be a matter of concern because, unlike other GUDs, genital herpes cannot be totally cured and asymptomatic shedding of herpes virus will not only increase it's (herpes) transmission but also increases HIV transmission.
\end{abstract}

Keywords: STD clinic attendees, Genital Ulcer Diseases, Prevalence.

\section{Introduction}

STDs have a tremendous impact not only on the individual affected but also on his family and also on the society and the nation as they may cause health problems like infertility, ectopic pregnancy, pelvic inflammatory disease, malignancies, abortions, preterm deliveries, neonatal morbidities thus making the policy makers invest a large amount on the families affected. STDs mainly manifest as GUDs, Genital discharges or Genital growths. GUDs are mostly caused by Syphilis, Chancroid, Donovanosis, LGV and Genital herpes.

Syphilis: It is caused by Treponema pallidum sub species pallidum, a micro organism belonging to the family spirochaetaceae. A chancre develops at the site of inoculation after an incubation period ranging from 1090 days (average of 21 days) depending on the amount of inoculam ${ }^{1}$. Syphilis may be congenital or acquired. The most common mode of transmission of acquired syphilis is sexual transmission. In the first two years of acquisition of acquired syphilis, it can be transmitted and is known as Early Infectious Phase which is further divided into Primary stage, Secondary stage, Recurrent stage and Early latent stage. Late non infectious stage (after second year of acquiring the infection) is classified into Late latent stage and Tertiary stage. Cardio vascular syphilis and Neuro syphilis are considered as Tertiary stage by some and as Quarternary stage by some $^{2}$. The primary lesion mostly presents as a solitary, painless ulcer with button hole induration. Regional lymph nodes are involved within a week of appearance of primary lesion. They are mostly bilateral, non tender, discrete with a firm rubbery consistency. Increased severity of clinical manifestations and painful rather than painless ulcers due to secondary infection and shorter incubation period are noted in syphilis in HIV patients ${ }^{3}$. Investigations for primary syphilis include Dark Ground Investigation, Non specific serological tests like Venereal Disease Research Laboratory(VDRL) test and Specific serological tests like Treponema Pallidum Haem Agglutination test.

Chancroid: It is an acute but localized GUD caused by Haemophilus ducreyi, a gram negative bacillus with a short incubation period of 1-14 days ${ }^{4}$. It usually presents with single or multiple painful, soft ulcers which bleed easily on manipulation. Lymph node involvement occurs within a week which is usually unilateral and tender. Lymph nodes may suppurate and form unilocular abscess which may rupture. In HIV infected persons, there may be alterations in the clinical picture with extensive necrotizing ulcers and multiple or multilocular buboes and healing may be delayed ${ }^{5}$. Gram stain of the smear from the ulcer base reveals typical "school of fish 
or rail road track" appearance .But microscopy is only 50\% sensitive compared to culture and should not be relied upon solely in the routine diagnosis of chancroid ${ }^{6}$. A probable diagnosis is made if the patient has 1 or more painful genital ulcers with (1) no evidence of treponema pallidum infection by DGI of the ulcer exudates or by a serological test of syphilis performed atleast 7 days after the onset of ulcers and (2) the clinical presentation, appearance of genital ulcers and if present, regional lymphadenopathy, are typical for chancroid and (3) a test for Herpes Simplex Virus (HSV) performed on the ulcer exudates is negative ${ }^{7}$.

Donovanosis: It is a chronic granulomatous disease of the genito inguinal region. It is also called as Granuloma Inguinale(GI). It is caused by Calymmatobacterium granulomatosis. The exact incubation period is not certain. However it is reported to vary from 8 days to 3 months in different studies and may even extend up to 1 year $^{8}$. The initial lesion occurs as a papule which breaks down to form an ulcer with well defined edges with beefy red granulation tissue which characteristically bleeds on touch. Lymphatic involvement is not a common feature in GI unlike other STDs like LGV. However, in some patients, sub cutaneous swellings may occur in the inguinal regions and may break down to form typical granulomas, often called as Pseudobuboes since there is no lymphadenitis on microscopic examination ${ }^{9}$. The lesions when associated with HIV infection may be larger and extensive with a higher incidence of psuedobubo formation and extensive ulceration ${ }^{10}$. Diagnosis is mainly clinical. Tissue smear using Giemsa or Leishman stain is simple and specific by detecting Donovan bodies.

LymphoGranulomaVenereum(LGV): It is a STD of lymph nodes caused by Chlamidia trachomatis types $\mathrm{L}_{1}, \mathrm{~L}_{2}, \mathrm{~L}_{3}$. It is a chronic systemic disease that begins at the site of inoculation, usually the genitalia and advances via the lymphatics. The course of the disease is characterized by a Primary stage, a Secondary stage(Inguinal syndrome) and a Tertiary stage(AnoGenitoRectal syndrome) ${ }^{11}$. The incubation period is 7-12 days or longer. In majority of patients no primary lesion is observed ${ }^{12} \cdot 10-30$ days after the appearance of a transient painless ulcer, unilateral tender lymphadenopathy develops. If untreated, it suppurates with multilocular discharging sinuses. If femoral lymph nodes are also involved, they are separated from the enlarged inguinal lymph nodes by pouport's ligament producing the characteristic "Groove's sign of Greenblatt". Elephantiasis of the genitalia due to lymphatic obstruction may cause Esthiomene in females and Ram's horn penis or Saxophone penis in the males. In the HIV infected persons with LGV, the clinical features are rapid to develop and may be more severe and may require prolonged duration of treatment ${ }^{13}$. Diagnosis is by clinical examination and by serological tests by detecting the increase in the antibody titres.

Genital Herpes: It is caused by 2 serotypes of Herpes simplex virus (HSV), HSV-1 and HSV-2 and mostly of the two types, by HSV-2. HSV characteristically persists in the sensory nerve root ganglia after the primary infection, in a latent form and will cause recurrent episodes whenever triggered and reactivated by factors like stress and trauma. Incubation period varies from 3-14 days. Asymptomatic viral shedding is the main mode of transmission. Clinically it can be a First episode or a recurrent episode. First episode can be a True Primary episode ( in a non immune person ) or Non primary first episode( positive serological tests without earlier clinical manifestations but with earlier asymptomatic infection). In the first episode there will be associated systemic manifestations like fever and malaise. The lesions characteristically appear as vesicles on an erythematous base which rupture to form superficial ulcers. Multiple ulcers may coalesce to form ulcers with polycyclic margins. Inguinal lymph node involvement occurs in the $2^{\text {nd }}$ or $3^{\text {rd }}$ week and they will be tender. Non primary first episode tends to have comparatively less systemic features and the duration of the lesions will also be shorter. Recurrent episodes are milder than the primary episodes. Many with recurrent episodes will have prodromal features like tingling and numbness or pain in the site before the appearance of lesions. Atypical presentations of herpes genitalis in patients with HIV infection include deep progressive ulceration, hemorrhagic lesions, ecthyma like lesions, hyper keratotic verrucous lesions resembling condylomata and continous and prolonged viral shedding ${ }^{14}$. Genital herpes is mostly diagnosed by clinical features. Tzanck smear test helps in the diagnosis. Serological tests which detect antibodies to HSV are useful in the diagnosis when there are no active lesions or when there are atypical presentations.

HIV infection which was first noticed only in 1980s is presently sweeping across the globe without any racial or geographic or gender or age barriers with catastrophic health and financial consequences. There is a strong association between the occurrence of HIV infection and the presence of other STDs. During the last decade, from different epidemiological, clinical, Biological and invitro studies, overwhelming evidence has accumulated that both ulcerative and non ulcerative STDs promote HIV transmission by augmenting HIV infectiousness and HIV susceptibility via different biological mechanisms ${ }^{15}$.

\section{Objectives}

In this context, a study was undertaken in the department of DVL on the STD clinic attendees over a period of one year from 01-04-2015 to 31-03-2016. The objectives of the study are to know:

1) The prevalence of GUDs in both sexes of the STD clinic attendees and 
2) The prevalence of individual GUDs in these patients.

\section{Methodology}

All the patients who attended the STD clinic over a period of one year were registered and examined for GUDs after taking their consent. Those patients who refused to give their consent were excluded from the study. A detailed clinical examination was done and the necessary investigations were performed. The investigations included routine investigations like total red blood cell count, hemoglobin percentage, total leukocyte count, differential count, erythrocyte sedimentation rate and random blood sugar estimation and the specific tests like HIV screening, VDRL test, Smear for gram stain and Leishman stain and Tzanck smear test and biopsy in needed cases. An appropriate diagnosis was made. The percentages were calculated. Wherever needed,, statistical significance was calculated using chi square test with yate's correction. The results were analysed and compared with similar studies.

\section{Results}

A total of 7925 STD clinic attendees were enrolled in the study. 1977 of them were males and 5948 were females (table 1, figure 1). Out of the total 7925 STD clinic attendees, 204 were diagnosed to be suffering with GUDs. Of them, 137 were males and 67 were females (table 1, figure 1). Out of the total 204 GUD patients, those suffering with various GUDs are as follows (table 2, figure2): 11 patients were suffering with primary syphilis of which 9 were males and 2 were females. Chancroid is seen in 13 patients of which 9 were males and 4 were female patients. No patients were diagnosed to be suffering with GI. 2 patients were diagnosed to be suffering with LGV and of them I is a male and 1 is a female patient who is having esthiomene. 169 patients were suffering with genital herpes of which 109 were males and 60 were female patients. 9 male patients were found to be suffering with non specific genital ulcers (NSUlc).

\section{Discussion}

Although there are many studies on the prevalence of individual GUDs, very few Indian studies were found in relation to the prevalence of all the GUDs in STD clinic attendees in India. In a study conducted by Sumathi Muralidhar et $\mathrm{al}^{16}$, the prevalence of GUDs is $7.45 \%$. In contrast to their study, in our study the prevalence is only $2.57 \%$ but the prevalence rate in male GUD patients of the total GUD patients is similar in both studies with $67.15 \%$ in our study and $66.7 \%$ in their study. Out of the total 1977 STD clinic male attendees, 137 were diagnosed to be suffering with GUDs which amounts to $6.93 \%$. Based on the chi square test with yate's correction, 2 tailed $p$ value is less than 0.0001 which is extremely statistically significant. Out of the total 5948 STD clinic female attendees, only 67 that is $1.13 \%$ were suffering with GUDs. Though this appears to be a small proportion, statistically it is significant as per Chi square test with yate's correction.

When individual GUDs were analysed, genital herpes is the leading cause of GUDs in our study (table 2 , figure 2). This is similar to many other studies ${ }^{17,18}$. Similar views were expressed by other authors also ${ }^{19,20}$. Chancroid used to be the leading cause of GUDs in the past as observed in some studies ${ }^{21}$, but recent studies have revealed a changing pattern of GUD in that GUD attributable to HSV-2 is increasing while that due to H.ducreyi is decreasing in many areas ${ }^{22}$. Chancroid is the second leading cause of GUD in our study with $6.37 \%$ of the total GUD patients suffering from it. This is similar to $8 \%$ observed in a study by Kumar B, Handa S et $\mathrm{al}^{23}$. Syphilis, one of of the leading causes of GUDs till 1990s as observed in many studies ${ }^{24,25}$, is on a decline. In a study by Risbud $\mathrm{A}_{\text {et }} \mathrm{al}^{26}$, syphilis is responsible for $10 \%$ of the cases while in our study, it is responsible only for $5.40 \%$ of the total GUD cases.LGV is less common than other STDs and recently a decreasing trend is observed $^{27,28}$. In similarity to these studies, only 2 cases of LGV were encountered in our study but classically with groove's sign in a male patient and with esthiomene in a female patient. In India the reported prevalence ranges from $0.2 \%$ to $11.5 \%{ }^{29}$. In correlation with this study, it is only $0.98 \%$ in our study. Donovanosis (GI) was claimed to be endemic in parts of India and South Africa ${ }^{30}$. But in our study which span over a period of one year, we did not see even a single case of GI. An explicit decline in the prevalence of the disease from $6.3 \%$ $(1977-85)$ to $0.77 \%$ (1993-99) has been pointed out in another study ${ }^{31}$. Perhaps it might had been further declined in this decade becoming almost extinct as is seen in our study.

\section{Photographs}




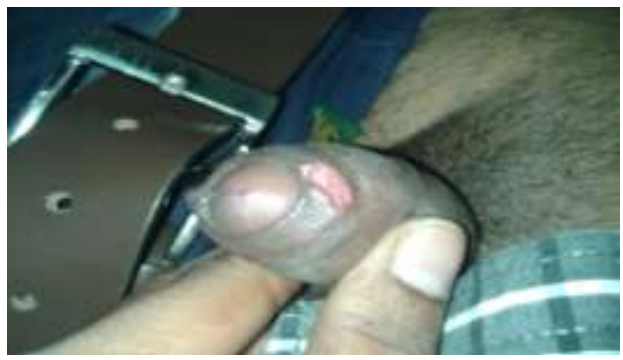

Primary Syphilis

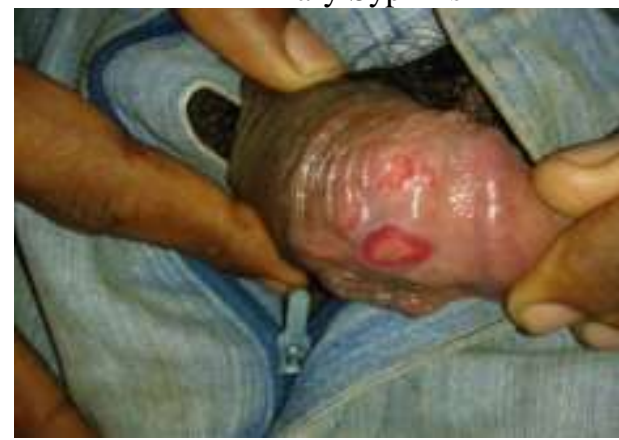

Genital Herpes

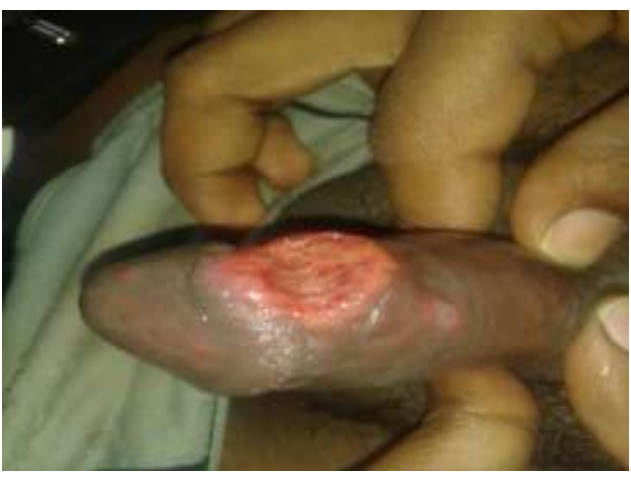

Non Specific Ulcer

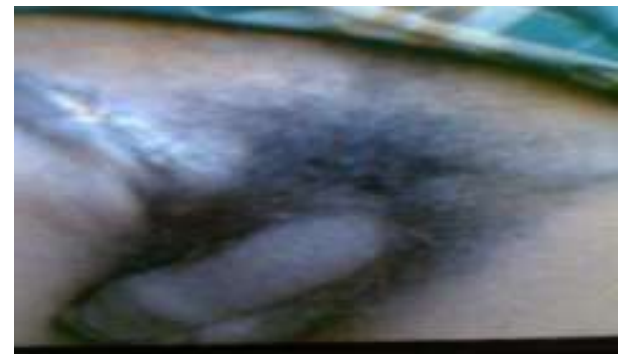

LGV

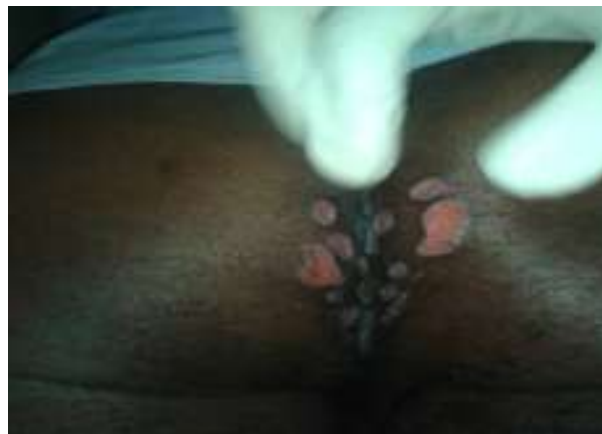

Genital Herpes

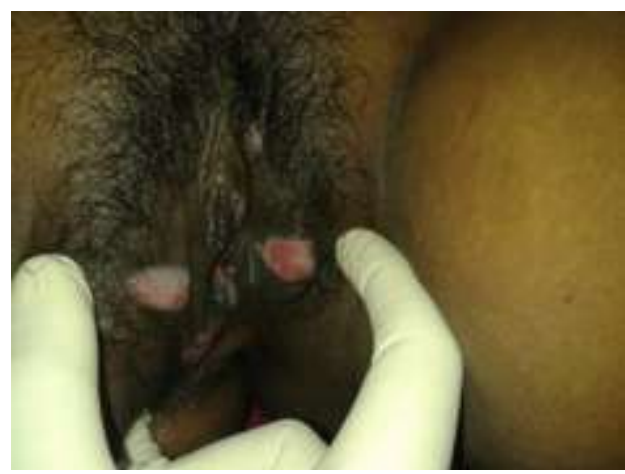

Chancroid

VII. Conclusion

Our study has certain limitations in that it could not utilize certain latest investigations like Polymerase Chain Reaction test and Antibody detection test etc which give sensitive and specific results for some GUDs like Chancroid, LGV and GI due to unavailability in our hospital. But at the same time, our study, with the available resources has clearly shown that the prevalence of GUDs is on a decline which is a welcome sign. The prevalence of syphilis and chancroid is decreasing and the prevalence of LGV is very minimal and GI is not at all found in our study. This decline in the prevalence of bacterial GUDs is a welcome note but at the same time, the viral GUD, genital herpes is on a rise as seen in our study with a Lion's share of $82.84 \%$ of the total GUDs. This is an alarming sign as the current treatment modalities of genital herpes cannot eradicate the latent virus. The asymptomatic viral shedding of HSV will not only increase the transmission of the HSV to the partners but also will increase the risk of transmission and acquisition of HIV infection. So, in order to decrease the incidence of HIV infection, the policy makers should concentrate more on the viral STDs and emphasize more on the prevention modalities like safer sex practices as the viral STDs like genital herpes cannot be totally cured.

Table1:- Sexual Distribution of total STD Patients and GUD Patients

\begin{tabular}{|c|c|c|c|}
\hline & $\begin{array}{l}\text { STD Patients (including } \\
\text { GUD) }\end{array}$ & GUD Patients & Percentage \\
\hline Total & 7925 & 204 & 2.57 \\
\hline Male & 1977 & 137 & 6.93 \\
\hline Female & 5948 & 67 & 1.13 \\
\hline
\end{tabular}


Table2:- Distribution of various GUDs

\begin{tabular}{|c|c|c|c|c|}
\hline Type of GUD & Male & Female & Total & Percentage \\
\hline Primary syphilis & 9 & 2 & 11 & 5.40 \\
\hline Chancroid & 9 & 4 & 13 & 6.37 \\
\hline GI & $\mathrm{X}$ & $\mathrm{X}$ & $\mathrm{X}$ & $\mathrm{X}$ \\
\hline LGV & 1 & 1 & 2 & 0.98 \\
\hline Genital herpes & 109 & 60 & 169 & 82.84 \\
\hline N S Ulc. & 9 & $\mathrm{X}$ & 9 & 4.41 \\
\hline
\end{tabular}

Figure 1:- Sexual Distribution of STD patients and GUD patients

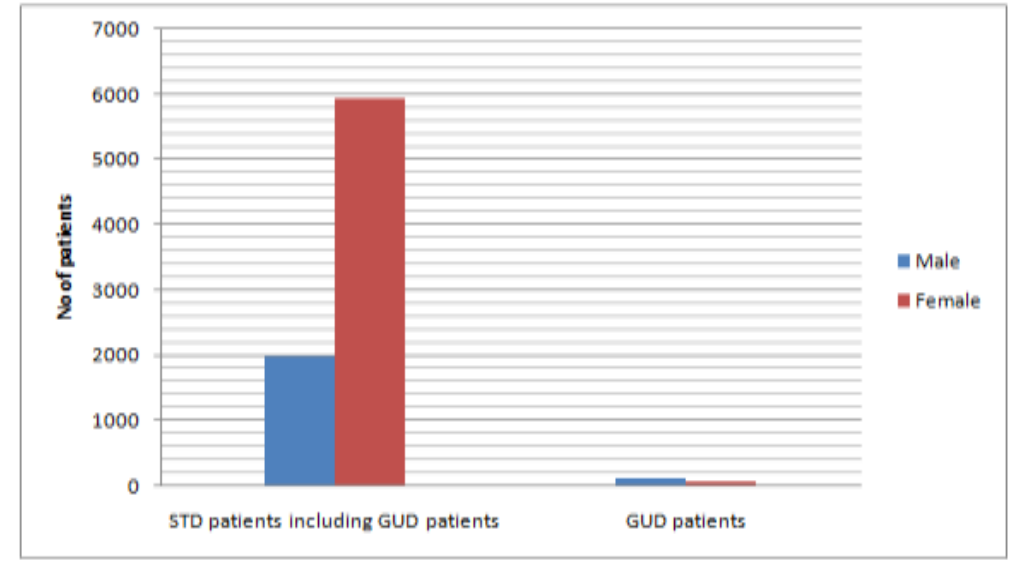

Figure2:- Distribution of various GUDs

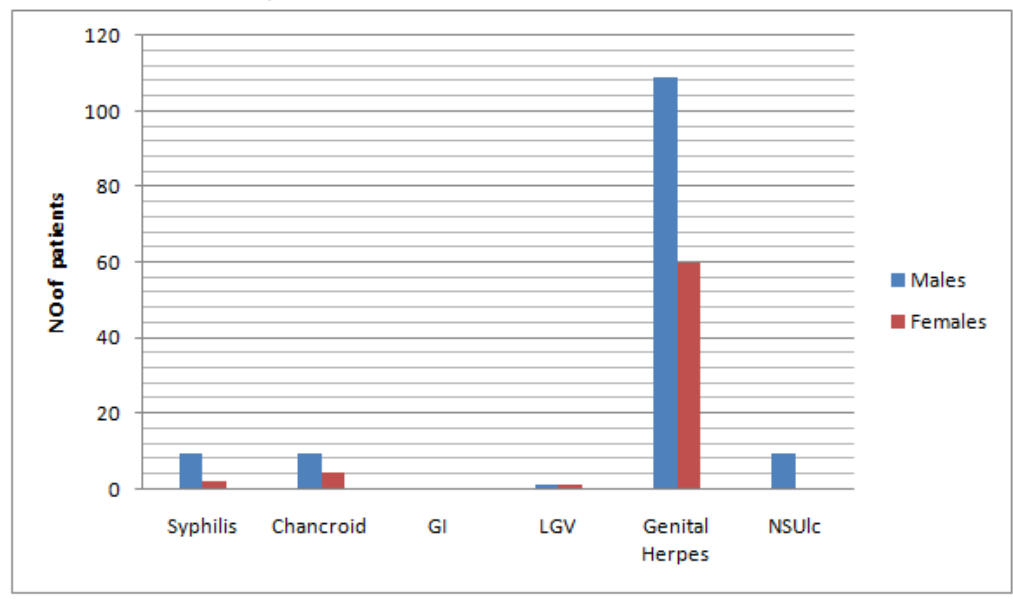

\section{References}

[1] Morton RS. The treponematoses. In: Rook A, Wilkinson DS, Ebling FJG, Champion RH, editors. Text book of dermatology. $4^{\text {th }}$ ed. London; Blackwell; 1986. P.839-83.

[2] King A, Nicol C. Syphilis. In: Venereal diseases. $3^{\text {rd }}$ ed. London; ELBS and Bailliere Tindall; 1975. P.7.

[3] Turbadkar D, Mathur M, Gaikwad S. Prevalence of syphilis among HIV sero reactive patients. Indian J Sex Transm Diseases. 2007; 28:91-93.

[4] Felman YM, Nikitas JA. Sexually transmitted diseases: Update on chancroid. Cutis. 1983; 602,607-8.

[5] Balachandran C, SatishPai B. Chancroid. In: Sharma VK, editor in chief. Sexually transmitted diseases and AIDS. $1^{\text {st }}$ ed. New Delhi; Viva books pvt ltd., 2003. p.219.

[6] Lewis DA. Tests for chancroid. Sex Transm Infect. 2000; 76: 137-41.

[7] Centers for Disease Control and Prevention; Workowski KA, Berman SM. Sexually Transmitted Diseases Guidelines 2006.MMwr Recomm Rep. 2006; 55 (RR-11) : 1-94.

[8] Rajam RV, Rangaiah PN. Donovanosis. WHO Monograph series. No.24; 1954.

[9] Greenblatt RB, Pund ER, Snaderson ES, et al. Management of chancroid, granuloma inguinale and lymphogranuloma venereum in general practice. U.S. Department of Health, Education and Welfare, Public Health Service No.255, Washington.

[10] Jamkhedkar PP, Hira SK, Shroff HJ, Lanjewar DN. Clinicoepidemiological features of granuloma inguinale in era of acquired immunodeficiency syndrome. Sex Transm Infect. 1998; 25: 196-200.

[11] Faro S. Lymphogranuloma venereum, chancroid, granuloma venerale. Obstrt Gynaec Clin North Am. 1989; 10: 517-30.

[12] Rajam RV, Rangaiah PN. Lymphogranuloma venereum. Indian J Dermatol. 1955; 21 suppli.1, No.4: 1-65.

[13] Czelusta A, Yen-Moore A, Vanderstraten M, Carrasco D, Tyring SK. An overview of sexually transmitted diseases in HIV infected patients. J Am Acad Dermatol. 2000; 43: 409-432.

[14] Sundharam JA. Herpes genitalis. In: Sharma VK, editor in chief. Sexually transmitted diseases and AIDS. $1^{\text {st }}$ ed. New Delhi; Viva books pvt.ltd.,2003. P.264. 
[15] Fleming DT, Wasserheit JN. From epidemiological synergy to public health policy and practice : the contribution of other sexually transmitted diseases to sexual transmission of HIV infection. Sex Transm Inf. 1999; 75: 3-17.

[16] Sumathi muralidhar, Richa Talwar, Deepa Anil Kumar, et al., "Genital Ulcer Disease : How Worrisome Is It Today ? A Status Report from New Delhi, India”, Journal of Sexually Transmitted Diseases, Vol.2013, Article ID 203636, 8 pages, 2013, doi: $10.1155 / 2013 / 203636$.

[17] Tariq A, Ross JDC. Viral sexually transmitted infections : current management stratagies. J Clin Pharm Ther. 1999; 24: 409-14.

[18] Mindel A. Genital herpes- how much of a public health problem. Lancet. 1998; 2351 (suppl.3) : 16-18.

[19] Gupta S, Kumar B, Genital herpes simplex infections. In: Kumar B, Gupta S, editors. Sexually transmitted infections. $1^{\text {st }}$ ed. NewDelhi; Elsevier; 2005. P.191-214.

[20] Habif TP. Sexually transmitted viral infection. In: Habif TP, editor. Clinical dermatology. $4^{\text {th }}$ ed. Edinburgh; Mosby; 2004. P.34655 .

[21] Reddy BSN, Nanda Soni. Chancroid. In: Kumar B, Gupta S, editors. Sexually transmitted infections. $1^{\text {st }}$ ed. NewDelhi; Elsevier; 2005.p.376-86.

[22] Sharma VK, Khandpur S. Changing patterns of sexually transmitted infections in India. Natl Med J India. 2004; 17:310-19.

[23] Kumar B, Handa S, Malhotra S. Changing trends in sexually transmitted diseases. Indian J Sex Transm Dis, 1995; 16:24-27.

[24] Urmil AC, DattaPK, Basappa K, Ganguly SS. A study of morbidity pattern among prostitutes attending a municipal clinic, Pune. J Indian Med Assoc. 1981; 83:29-31.

[25] Kapur TR. Pattern of sexually transmitted diseases in India. Indian J Dermatol Venereol Leprol. 1982; 48:23-24.

[26] Risbud A, Chan-Tack K, Gadkari D, et al. The etiology of infection, genital ulcer disease by multiplex polymerase chain reaction and relationship to HIV infection among patients attending sexually transmitted diseases clinics in Pune, India. Sex Transm Dis. $1999 ; 26: 55-62$.

[27] Jaiswal AK, Bhushan B. Pattern of sexually transmitted diseases in North Eastern India. Indian J Sex Transm Dis. 1994; 15:19-20.

[28] Reddy BSN, Garg BR, Rao MV. An appraisal of trends in sexually transmitted diseases. Indian J Sex Transm Dis. 1993; 14:1-4.

[29] Kapur TR. Pattern of sexually transmitted diseases in India. Indian J Dermatol Venereol Leprol. 1982; 48: 23-24.30. O'Farell N. Donovanosis. Sex Transm Infect. 2002; 78: 453-57.

Address for correspondence: Dr. DSS Srinivas Prasad, Dept. of DVL, Guntur Medical College, Guntur-522001, Andhra Pradesh, India. 